\title{
Traumatic grief in young people in Sub-Saharan Africa: a scoping review
}

\author{
This article was published in the following Dove Press journal: \\ Nursing: Research and Reviews \\ 16 July 2015 \\ Number of times this article has been viewed
}

\author{
Holly Taggart ${ }^{\prime}$ \\ Sheila Greatrex-White ${ }^{2}$ \\ 'Mental Health Commission, \\ CentreForum, Westminster, UK; \\ ${ }^{2}$ School of Health Sciences, Queen's \\ Medical Centre, University of \\ Nottingham, Nottingham, UK
}

Aim: To identify relevant and pertinent themes and interventions within the literature relating to childhood traumatic grief, in order to provide a sound background of evidence for further research and service development.

Background: Childhood traumatic grief is caused when a significant person in a child's life dies under circumstances that they perceive to be traumatic. This can leave a child unable to return to the same level of physical and emotional functioning that he or she had prior to the death occurring. In Sub-Saharan Africa, there is an increased risk for childhood traumatic grief due to a high prevalence of orphanhood, environmental stressors, stigma, and abuse. This can have detrimental effects upon mental health.

Methods: The review followed the York methodology: identifying the purpose and agreeing on the strategy beforehand; identifying relevant sources/studies; selecting the studies; charting the data; and collating, summarizing, and reporting results.

Results and discussion: Interventions identified to prevent and/or manage traumatic grief included narrative exposure therapy, psychotherapy, mentoring, peer-group support, psychosocial support, a grief and loss therapy session, and memory boxes. Mental health remains neglected within service and policy development as well as in global health spending. The average amount expended on mental health services per person per year in low-income countries is less than $\$ 0.25$. Only $36 \%$ of people in low income countries are covered by a mental health policy, compared with $92 \%$ in high income countries.

Limitations: The sixth stage of the York methodology was omitted. Only papers written in English were included in the review.

Conclusion: Childhood traumatic grief in young people is an important issue within SubSaharan Africa and has relevance to the global mental health agenda.

Implications for nursing and health policy: With the HIV/AIDS pandemic continuing to threaten and the number of orphans estimated to rise, a number of recommendations developed from the literature are suggested.

Keywords: childhood traumatic grief, HIV/AIDS, interventions, mental health, orphans, scoping review, Sub-Saharan Africa, young people

\section{Aim, rationale, and background}

We aimed to identify relevant and pertinent themes within the literature relating to childhood traumatic grief (CTG) in order to provide a background of evidence for further research and service development. Both authors have been collaborating with colleagues in Sub-Saharan Africa (SSA) for a number of years and have come to understand some of the issues relating to CTG. This review encompasses how populations within SSA view and experience death, orphanhood, and traumatic grief.
Correspondence: Sheila Greatrex-White School of Health Sciences, B Floor, Room B62, Queen's Medical Centre, University of Nottingham, Nottingham NG7 2HA, UK Tel +44 II 58230960

Email sheila.greatrex-white@nottingham. ac.uk submit your manuscript | www.dovepress.com

Dovepress

http://dx.doi.org/1 0.2147/NRR.S80142 
CTG is relatively underresearched in spite of its importance globally, and specifically to SSA. ${ }^{1}$ It has been estimated that 14 million children have been singly or doubly orphaned in SSA due to human immunodeficiency virus (HIV) acquired immunodeficiency syndrome (AIDS), malaria, and tuberculosis-related deaths. ${ }^{2}$ Furthermore, Thupayagale-Tshweneagae and Mokomane ${ }^{3}$ argue that the needs of adolescents orphaned by AIDS are seldom recognized or adequately addressed in policy and programs. According to Cluver and Gardner ${ }^{4}$ and Thupayagale-Tshweneagae et al, ${ }^{5}$ orphanhood increases the probability of a child developing posttraumatic stress disorder and depression. Despite these risk factors, mental health services remain underdeveloped and have been criticized for being unable to meet the needs of communities, particularly as mental health policies generally are limited. ${ }^{6}$ This review is particularly relevant for service providers, researchers, and stakeholders working in the field of child development, child and adolescent mental health, and global mental health.

We present key definitions, the methods used in the scoping review, and the themes that emerged from the literature. The themes are: traumatic grief in context; the significance to SSA of CTG; the influence of orphanhood upon mental health; community attitudes toward HIV/AIDS and orphanhood; cultural influences upon the expression of grief; diagnosing and managing CTG and its comorbidities; service and policy development in SSA; and formal and informal service provisions.

\section{Key definitions}

\section{Traumatic grief}

The terms "traumatic grief", "complicated grief", and "prolonged grief" are used interchangeably within Western literature; within this review, the term "traumatic grief" was chosen. CTG occurs when a significant person in a child's life dies under circumstances that the child perceives to be traumatic. ${ }^{7}$

\section{Young people}

A young person was considered to be between the ages of 0-26 years.

\section{Sub-Saharan Africa}

Sub-Saharan Africa includes countries in western, eastern, and southern Africa, as defined by the United Nations. ${ }^{8}$

\section{Formal sector}

In this review, the formal sector included any statutory health service funded by the Ministry of Health.

\section{Informal sector}

In this review, the informal sector included any service that provides health and/or social care that is not funded through a statutory organization.

\section{Methodology and methods}

A scoping review was chosen, as it is primarily a way of summarizing a range of evidence in order to convey the breadth and depth of a field; it allows gaps within the literature to be identified and suggests future areas of research. ${ }^{9}$ This can increase awareness of a particular topic, which is a key issue for CTG. Importantly, scoping reviews are not intended to assess the quality of the existing literature although we comment briefly on this. We did not exclude studies on grounds of quality, not least because it would have eliminated the majority of studies but also because, as Sandelowski ${ }^{10}$ points out, there are wide variations in concepts of "the good" and in what constitutes "quality criteria". A systematic scoping process employing the first five stages of the York methodology was used. ${ }^{11}$ This included: identifying the purpose of the review and agreeing on the strategy beforehand; identifying relevant sources/studies; selecting the studies; charting the data; and finally, collating, summarizing, and reporting results. Both authors were involved in all stages, including independent abstract review, full article review, and data charting decisions. We had made provision to call upon colleagues willing to arbitrate where disagreement occurred, but this was not necessary. Although a sixth stage in the York methodology involves consulting with stakeholders, this stage was omitted even though some would argue that doing so might compromise methodological rigor. ${ }^{12}$

\section{Search terms}

The terms "grief", "bereavement", and "mourn" were chosen to relate to the traumatic grief aspect of the review. The term "traumatic" was not included because it did not yield any results. The terms "child", "adolescent", "young people", and "youth" were included to represent the population group. The term "Africa" was chosen to include all studies within African countries. "Sub-Saharan Africa" was not used as it did not yield any results, but specific databases allowed synonyms of "Africa" to be included. The term "intervention" was not incorporated as it did not yield any results.

\section{Sources searched}

A total of six databases and eight global networks were searched, including: Allied and Complementary Medicine 
(AMED), Applied Social Scientists Index and Abstracts (ASSIA), the Cumulative Index to Nursing and Allied Health Literature (CINAHL), the Excerpta Medica dataBASE (EMBASE), the Medical Literature, Analysis and Retrieval System Online (MEDLINE), PsycINFO, HOPEHIV, Orphans and Vulnerable Children (OVC), the Population Council, Save the Children, the Tropical Health Education Trust (THET), the Joint United Nations Programme on HIV/AIDS (UNAIDS), the United Nations Children's Fund (UNICEF), and the World Health Organization (WHO) (see Table 1).

Qualitative or quantitative empirical studies from any time period (up until March 2013) were included, as well as unpublished documents ("grey literature"), book chapters, reports, conference abstracts, theses, and dissertations, as this enabled all data relating to the topic to be identified and bias to be minimized. References and bibliographies of all included studies were searched for key terms in order to identify other studies that could not be accessed through databases. Studies presented in a language other than English were excluded due to time and financial constraints of translating papers, although none were identified.

\section{Results and discussion \\ Childhood traumatic grief in context}

CTG is a form of psychological trauma that differs from a typical grief response. It occurs when a significant person in a child's life dies under circumstances that the child perceives to be traumatic. ${ }^{7}$ This initiates a grieving process as well as a trauma response. ${ }^{13}$ Prigerson ${ }^{14}$ defines traumatic grief as an inability to return to the level of physical and emotional functioning that one had prior to a death. Children may also experience secondary loss, such as changes to their lifestyle. ${ }^{15}$ While the grieving process is often misunderstood in terms of the child's level of understanding, Corr and Balk ${ }^{15}$ argued that very young children also will feel loss, even if they do not know the reason for it. Therefore, as children react to death, grief can manifest psychologically, physically, socially, or spiritually over different periods of time.

Beveridge $^{16}$ reported that survival instincts are utilized during traumatic experiences, resulting in a psychophysiological response. Following a traumatic event, children may present with symptoms concurrent with Type I or Type II trauma. ${ }^{17}$ Type I trauma includes symptoms of denial, numbing, dissociation, and anger and is more prominent after a single unanticipated event has occurred. Type II trauma includes symptoms of detailed recall and misperceptions of an event that are often caused by recurrent exposure to traumatic circumstances, suggesting that children are able to anticipate a trauma and adapt. Terr ${ }^{17}$ also suggests that some children present with mixed type trauma (symptoms of Type I and Type II trauma), particularly if the trauma has involved an unexpected death. Hence, bereaved children may show symptoms concurrent with Type I trauma initially and over time develop symptoms of Type II trauma.

Traumatic grief is often associated with posttraumatic stress disorder, which is a term used to describe the reactions that manifest following a traumatic event that induces clinically significant distress or impairment. This may mani-

Table I Sources searched for formal and informal service provision

\begin{tabular}{|c|c|c|c|c|c|c|}
\hline & Source & $\begin{array}{l}\text { Initial } \\
\text { search }\end{array}$ & Accepted & $\begin{array}{l}\text { Excluded at } \\
\text { a later date }\end{array}$ & $\begin{array}{l}\text { Forward and reverse } \\
\text { citation tracking }\end{array}$ & Included \\
\hline \multirow[t]{6}{*}{ Database } & AMED & 8 & I & I & 0 & 0 \\
\hline & ASSIA & 7 & 0 & 0 & 0 & 0 \\
\hline & CINAHL & 0 & 0 & 0 & 0 & 0 \\
\hline & EMBASE & 70 & 3 & 2 & 0 & I \\
\hline & MEDLINE & 63 & 4 & 3 & 2 & 2 \\
\hline & PsyclNFO & 150 & 4 & 3 & 0 & I \\
\hline \multirow[t]{8}{*}{ Global network } & HOPEHIV & 14 & 0 & 0 & 0 & 0 \\
\hline & ovc & 5 & 1 & I & 0 & 0 \\
\hline & Population Council & 39 & 11 & 7 & 0 & 4 \\
\hline & Save the Children & 22 & 0 & 0 & 0 & 0 \\
\hline & THET & 17 & 1 & 1 & 0 & 0 \\
\hline & UNAIDS & 357 & 0 & 0 & 0 & 0 \\
\hline & UNICEF & 24 & 0 & 0 & 0 & 0 \\
\hline & WHO & 6 & 0 & 0 & 0 & 0 \\
\hline Further search & & - & - & - & 3 & 3 \\
\hline Total & 14 & 782 & 25 & 18 & 5 & 11 \\
\hline
\end{tabular}

Abbreviations: AMED, Allied and Complementary Medicine Database; ASSIA, Applied Social Scientists Index and Abstracts; CINAHL, Cumulative Index to Nursing and Allied Health Literature; EMBASE, Excerpta Medica dataBASE; MEDLINE, Medical Literature, Analysis and Retrieval System Online; OVC, Orphans and Vulnerable Children; THET, Tropical Health Education Trust; UNAIDS, Joint United Nations Programme on HIVIAIDS; UNICEF, United Nations Children's Fund; WHO, World Health Organization. 
fest in children as repetitive reenactment of the trauma, nightmares, and disorganized or agitated behavior. ${ }^{18}$ Posttraumatic stress disorder is categorized as a mental disorder but can develop in people without prior mental health problems. Alternatively, these disorders can exist comorbidly with anxiety, attention deficit hyperactivity disorder, conduct disorder, and substance misuse, although these are often misdiagnosed as personality disorder, dissociative disorder, or phobic disorder. ${ }^{17}$

Traumatic grief has also been associated with psychosis - a psychiatric diagnosis that typically manifests within adulthood. ${ }^{19}$ Ross ${ }^{20}$ developed the dissociation model, which suggests adults who have experienced a childhood trauma may present with symptoms similar to schizophrenia, although they are more likely to exhibit positive symptoms (hallucinations, delusional beliefs, thought disorders) than negative symptoms (poverty of speech, loss of motivation, blunted emotional affect). This implies that the traumatic event is a key factor of psychosis and that a diagnosis of dissociative psychosis as opposed to schizophrenia may be more appropriate. However, as traumatic grief, posttraumatic stress disorder and psychosis are Western psychiatric definitions, the transferability of these concepts to other cultures and contexts is questionable. They also provide only one explanation of the causal relationship between childhood trauma and mental health problems. ${ }^{21}$ Overall, CTG is a relatively new concept within the literature, and further work is still developing. The nature of trauma research has also been questioned on ethical grounds, due to difficulties gaining informed consent from traumatized participants and the potential for generating further distress. ${ }^{22}$

\section{The significance of childhood traumatic grief in Sub-Saharan Africa}

In SSA in 2009, an estimated 14.8 million children (aged 0-17 years) had been orphaned due to AIDS, ${ }^{2}$ with this figure predicted to rise in future years. The death of a parent or parental figure is one of the most stressful events a child can experience, and AIDS-related deaths are associated with traumatic grief. ${ }^{23}$ If this occurs, there are pre-risk factors (previous life stressors), peri-risk factors (feeling loss of control), and post-risk factors (poor level of social support) that make a child more likely to experience mental health problems. $^{24}$

McDermott ${ }^{25}$ indicated that there is a culture of silence regarding death and dying in SSA in the days immediately following the death of a parent. This results in young people being discouraged from mourning the death of their parents externally. Wood et $\mathrm{al}^{26}$ reported similar findings within SSA. This can leave young people physically and emotionally unprepared for death. ${ }^{27}$ Van der Heijden and Swartz ${ }^{28}$ discussed an intervention that broke the cultural silence and allowed young people to remember and grieve. This intervention was conducted by researchers from SSA, despite using an approach nonconcordant with cultural practices. This recognizes the importance of ensuring that local researchers are involved in service development to make sure that interventions are culturally sensitive, especially as expressions of grief can be different within Western countries ${ }^{29}$ although, as Van der Heijden and Swartz ${ }^{28}$ acknowledged, the social constructs of grief are yet to be explored.

Nevertheless, even when many risk factors are present, it is evident that some children remain resilient. Masten ${ }^{30}$ suggested that disadvantaged children are more likely to be resilient to bereavement, particularly as they are likely to have experienced trauma historically. ${ }^{17}$ Although risk factors and resilience across different cultures and contexts have been widely researched, and while ethnographic data suggests that children in SSA and children residing in high-income countries have similar prevalence rates of mental health problems, ${ }^{31}$ research into the prevalence of CTG remains limited, particularly within low-income countries, making it difficult to assess the extent of this phenomenon within populations in SSA.

\section{The influence of orphanhood upon mental health}

The relationship between orphanhood and mental health can be categorized into contributing risk factors and presentation of mental distress. Gilborn et $\mathrm{al}^{32}$ identified contributing risk factors as illness within the family, limited access to healthcare, and stigma and social isolation. This is in addition to molestation and rape, which were associated with depression and anxiety. Another risk factor reported within the findings involved community and sexually based violence. ${ }^{32}$ Specifically in South Africa, there is an exceptionally high rate of violent death. Despite these traumatic experiences, young people who have experienced many stressors were more likely to say they were able to cope with difficulties, compared with people who had experienced the least trauma. The orphan crisis can exacerbate problems within resource-poor communities, as $40 \%$ of HIV positive parents are fostering at least one child who is not their own. ${ }^{33}$ This could portend pretraumatic risk factors. Gilborn et $\mathrm{al}^{32}$ also reported the risks associated with being an orphan of AIDS. This is because AIDS orphans have a higher propensity for negative mental health factors 
compared with those orphaned from other causes. ${ }^{34}$ Risk factors for the development of mental health problems have been widely researched within the literature, although risk factors and resilience are individual experiences and do not necessarily apply to whole populations.

Orphaned young people will often present with symptoms of anxiety, including feeling worried and overwhelmed. Mental distress might also present through anger, fear, guilt, and somatizations (physical symptoms from psychological cause). This includes headaches, nightmares, fatigue, and poor concentration. ${ }^{35}$ Anxiety can also tend to manifest with an external focus, particularly upon social and economic contexts. By comparison, other young people will internalize anxiety, potentially leading to depression and lowered self-esteem. Conflicting evidence suggests AIDS orphans are more likely to internalize problems. ${ }^{36}$ Hopelessness is also reported in some young people, although others are able to display some signs of optimism and report feeling happier. ${ }^{28,32}$ Within the intervention studies, older children tended to report greater confidence and self-esteem despite greater exposure to traumatic events. There does, however, appear to be a sex difference, with females reporting greater exposure to death, traumatic events, and daily stress compared with males. Consequently, females were more likely to show higher levels of psychosocial distress and lower psychosocial wellbeing than males.

\section{Community attitudes toward HIV/AIDS and orphanhood in SSA}

Denis and Makiwane ${ }^{37}$ found that adults need to have a trusting relationship with members of a community before disclosing HIV status. Gilborn et $\mathrm{al}^{32}$ acknowledged that the majority of parents stated they have not talked directly to their children about HIV/AIDS in spite of older children viewing disclosure positively. One reason for reluctance to disclose HIV status is stigma, something directed toward women more than men. Even though HIV/AIDS is highly prevalent across SSA, it remains a taboo subject. Stigma is associated with negative mental health. ${ }^{38}$ However, two interventions report a reduction in stigmatizing behavior through education. ${ }^{39,40}$

Kayombo et $\mathrm{al}^{35}$ found that young people who were orphaned experienced stigma from friends, family, and their community. Some children were also rejected by their relatives after the death of their parents for fear they might infect their own children. This could predispose to young people becoming homeless after they are orphaned. Idoniboye $^{41}$ suggested that behavioral problems associated with orphanhood can make it difficult for young people to be placed into a family, but conflicting research indicates that many standby guardians care for a child who is not their own. ${ }^{33}$ Whereas some young people do not receive the support they need from adults and peers in order to deal with their traumatic stressors, one study found that $88 \%$ said they could ask an adult for help if they needed it and 90\% had enjoyed spending time with their peers in the past month. ${ }^{32}$ Feeling supported by family, peers, and the wider community is a protective factor that reduces the probability of developing traumatic grief reactions or mental health problems. ${ }^{42}$ Attitudes about HIV disclosure and orphanhood need to be addressed, as stigma and bullying increases the likelihood of a young person developing a psychological disorder. ${ }^{43}$ While acknowledging these studies, the literature pertaining to public perceptions of HIV/AIDS and orphanhood within SSA remains limited.

\section{Cultural influences upon the expression of grief in SSA}

One risk factor for traumatic grief involves the understanding and perception of death and dying, which differs among cultures and contexts. ${ }^{44}$ Gilborn et $\mathrm{al}^{33}$ suggested that for parents diagnosed with AIDS, the greatest concern is to ensure that their children have access to education, food, and clothing; emotional suffering was the least concerning factor expressed. This emphasizes how material needs are prioritized within SSA and aligns with Opperman, ${ }^{45}$ who suggested that the risk for developing traumatic grief was elevated when material wealth was threatened. An intervention involving traditional healers, who would comfort orphans by telling them stories about other orphans who had managed to cope and gain achievement in life, allowed children space to remember and grieve. Although emotionally challenging, it was positive in that it encouraged the children to build a more optimistic attitude toward life as orphans. ${ }^{28}$ It is acknowledged, however, that the social constructs of grief in the context of SSA are yet to be explored.

\section{Diagnosing and managing childhood traumatic grief and comorbidities}

Prigerson et $\mathrm{al}^{46}$ make the observation that traumatic grief is not established as a clinical diagnosis within the Diagnostic and Statistical Manual of Mental Disorders, fourth edition. Melhem et $\mathrm{al}^{47}$ suggest that this reflects the interlinked relationship between traumatic grief and the two main psychiatric sequelae, posttraumatic stress disorder and depression, despite evidence to suggest they are independent from each 
other. As a result, guidelines on the management of traumatic grief are less clear within the literature, and its absence from the manual makes it difficult for health workers to identify and manage the issue. It is of interest to note that amongst the changes in the most recent update of the manual (the Diagnostic and Statistical Manual of Mental Disorders, fifth edition, published in May 2013), a new diagnosis of "persistent complex bereavement related disorder" is proposed, together with a new diagnostic category of "trauma and stressor related disorders". ${ }^{48}$ These changes are not without their critics.

The Mental Health Gap Action Programme, launched by the World Health Organization in 2008, provides a diagnostic and treatment algorithm for a range of mental health problems in nonspecialist primary care settings; in one subsection of the manual, health workers are advised to ask if a person has been exposed to extreme stressors such as bereavement. The recommended guidelines are to support culturally appropriate mourning; mobilize social networks; provide psychological first aid in cases of extreme distress; and address physical needs. Additionally, health workers are encouraged to identify any other mental health problems and follow the recommended treatment plan within that subsection. ${ }^{38}$ However, the Mental Health Gap Action Programme does not include specific information relating to the identification and treatment of traumatic grief in young people. This is an important omission because, although the majority of children who experience a traumatic event will demonstrate a typical trauma reaction, a minority of children will need psychological or pharmacological intervention. ${ }^{49}$

According to Dyregrov, ${ }^{50}$ who compared the processes in Europe and the United States for dealing with critical incident stress, debriefing should be the first intervention offered to a child after a traumatic event: this can be applied within different cultures and contexts and utilizes group therapy and leadership techniques. Following this, bereavementfocused cognitive-behavioral therapy can be implemented. In addition, general observations should be carried out for symptoms of traumatic grief, acute stress reaction, and posttraumatic stress disorder, although acute stress reaction and posttraumatic stress disorder should be managed before traumatic grief, but without avoiding discussion of the grief. ${ }^{19}$ If acute stress reaction or posttraumatic stress disorder is suspected, assessment tools can be utilized to make a formal diagnosis by measuring reactions to trauma ${ }^{51}$ and the severity of posttraumatic stress disorder symptoms. ${ }^{52}$ Interventions implemented to manage posttraumatic stress disorder include trauma-focused cognitive-behavioral therapy and eye movement desensitization and reprocessing. Cognitive-behavioral therapy is an intervention that encourages an individual to challenge their thoughts, feelings, and behaviors over a set period of time. ${ }^{53}$ Eye movement desensitization and reprocessing uses bilateral stimulation, mostly in the form of eye movements, to allow reprocessing of traumatic memories. ${ }^{54}$ This is achieved by focusing upon specific images, negative sensations, and associated cognitions, and desensitizing these memories by introducing positive sensations and cognitions. These interventions must be appropriate in terms of age and developmental understanding and incorporate sensitive language to explain about death and grief.

\section{Service and policy development in Sub-Saharan Africa}

Globally, mental health remains neglected within service development, ${ }^{55,56}$ policy development, ${ }^{57}$ and global health spending. ${ }^{58}$ In terms of policy and service development, the average money spent on mental health services in low-income countries is less than $\$ 0.25$ per capita per year. This compares to global spending within mental health services that equates to $\$ 1.63$ per person per year and mental health expenditure per capita that is more than 200 times greater in high-income countries than in low-income countries. ${ }^{55}$ In addition, only $36 \%$ of people residing in low-income countries are covered by a mental health policy, compared with $92 \%$ of people residing in high-income countries.$^{59}$ However, mental health is gaining status and credibility, with several low-income and middle-income countries now beginning to introduce policies and service provisions, ${ }^{60}$ albeit that the budget allocated to these provisions continues to remain disproportionate to the scale of the problem. ${ }^{58}$

Health worker shortages are a huge problem in SSA and can be attributed to a number of factors. Firstly, the existing work force is unable to work effectively, as $41 \%$ are estimated to be HIV positive. ${ }^{61}$ This is exacerbated by resource limitations. ${ }^{62}$ Secondly, many countries lack the financial means to train staff. ${ }^{63}$ Maj $^{64}$ suggested there is a need for psychiatrists to be trained to a higher standard, to cover a wider area of the population and to adhere to psychosocial interventions. Thirdly, it is common practice for healthcare professionals to work in affluent geographical areas where health needs are not as severe. ${ }^{63}$ Professional migration is often caused by push factors, including war, civil unrest, and economic deterioration, as well as pull factors, including salary, job satisfaction, and workplace environment. ${ }^{65,66}$ This has resulted in many professionals from SSA emigrating 
to more affluent countries, adding to the burden of worker shortages in their own country.

\section{Formal and informal service provisions and interventions}

\section{Specialist services}

Schaal et $\mathrm{al}^{67}$ evaluated the efficacy of narrative exposure therapy compared to interpersonal psychotherapy in a pilot randomized controlled trial with two groups of Rwandan genocide orphans. The outcomes measured included posttraumatic stress disorder and depressive symptomology. Narrative exposure therapy is a form of trauma-focused cognitive-behavioral therapy that is aimed at integrating emotional, cognitive, physiological, and sensory memories within the context of traumatic experiences. Interpersonal psychotherapy uses a series of techniques to manage posttraumatic stress disorder, depression, and interpersonal relationships and find ways of coping with these problems by helping individuals to gain insight into their difficulties. Participants in the narrative exposure therapy group received three individual therapy sessions and one grief session, while interpersonal psychotherapy participants also received four therapy sessions. At the 3-month posttest, no significant differences were found between any of the outcome measures, but at the 6-month follow up the proportion of participants in the narrative exposure therapy group who still had a diagnosis of posttraumatic stress disorder had decreased to $25 \%$, compared with $71 \%$ of participants in the interpersonal psychotherapy group who still had a diagnosis of posttraumatic stress disorder $(P<0.05)$. This study showed that narrative exposure therapy constituted an effective treatment for traumatized survivors with symptoms of posttraumatic stress disorder and depression and was more effective than interpersonal psychotherapy in reducing the severity of symptoms.

An intervention delivered at a specialist level was reported by McDermott, ${ }^{25}$ who evaluated the use of integrative longterm psychotherapy with an eleven-year-old girl from South Africa who had been abused and singly orphaned. This intervention was found to have a positive effect upon the child's mental health, as evidenced by a reduction in hypervigilance, an improvement in healthy play, and the development and maintenance of relationships with peers. In this study the intervention was delivered by a clinical psychologist, and in the study by Schaal et a ${ }^{67}$ both narrative exposure therapy and interpersonal psychotherapy were conducted by health professionals with a psychology background. While this is positive in ensuring that interventions are appropriate and evidenced based, it could also result in a lack of transferability to other contexts where health professionals are unavailable to implement these interventions. In both studies, interventions were confined to small participant numbers of 1-26 people. Before the results can be generalized, there is a need for larger-scale studies to be conducted in order to ascertain the effectiveness of the interventions on the wider population.

\section{Non-specialist services}

We found eight interventions that were delivered at a nonspecialist service level by nonhealthcare professionals. All of these interventions aimed to provide psychosocial support to orphans and vulnerable youths. Apicella et $\mathrm{al}^{40}$ evaluated a US-funded intervention program entitled "Reaching HIV/AIDS Affected People with Integrated Development Support (RAPIDS)", which incorporated psychosocial support for young people aged 10-24 years in Zambia. The program leader delivered this intervention, which aimed to provide emotional and spiritual counseling to vulnerable young people and chronically ill people. An improvement in school attendance, skills for work, and material needs and a decrease in stigmatizing behaviors was observed following the intervention.

Chitiyo et $\mathrm{al}^{68}$ evaluated the effectiveness of psychosocial support in improving school outcomes of children with special needs aged 10-14 years in Zimbabwe. Conducted by teachers at the school, this intervention included grief and bereavement counseling, ball games, and a buddy system and was considered to improve confidence, selfesteem, motivation, class work, and school attendance. Similarly, Gilborn et $\mathrm{l}^{32}$ compared the use of three intervention groups and a control group in orphans and vulnerable youths aged 14-20 years, also in Zimbabwe. These groups comprised: 1) youth exposed to community psychosocial support only; 2) youth exposed only to the Salvation Army Masiye Camp, a residential psychosocial support program; and 3) youth who attended Masiye Camp and went on to become a youth counselor or leader. The comparison group received none of the above. The community psychosocial support involved training teachers to recognize and respond to psychosocial distress among students; Kids' Clubs that met weekly for group recreation, socialization, and games; and group and individual counseling and problem solving. The Masiye Camp was a 10-day residential, faith-based program aimed at improving the psychosocial well-being of participants. The leader group consists of youth who attended Masiye Camp as campers and then became either Masiye Camp counselors 
or community psychosocial-support youth leaders. Males who participated in the community psychosocial program were more likely than their comparison-group peers to report self-confidence ( $85 \%$ vs $77 \%$, adjusted odds ratio $[\mathrm{AOR}]=1.85,95 \%$ confidence interval $[\mathrm{CI}]=1.03-3.33$; $P<0.05)$. However, no other associations were identified between the community psychosocial support group and the comparison group. Males who attended Masiye Camp were significantly more likely than comparison-group males to report feeling confident in the last month $(87 \%$ vs $77 \%$, AOR $=2.27,95 \% \mathrm{CI}=1.14-4.45 ; P<0.05$ ). For males, the leadership experience was strongly associated with self-confidence $(96 \%$ vs $77 \%$, AOR $=6.83$, $95 \% \mathrm{CI}=1.49-31.34, P<0.05)$.

Nelson et a $\mathrm{l}^{139}$ assessed the feasibility of training youth caregivers to provide HIV education and psychosocial support to orphans and vulnerable children aged 11-15 years in South African schools through counseling. This was found to improve the psychosocial wellbeing of participants through increased confidence and an improvement in school work.

Van der Heijden and Swartz ${ }^{28}$ evaluated a Vhutshilo intervention that consisted of 13 education sessions, one being a grief and loss session for young people aged 10-13 years living in resource-poor communities in South Africa. The aim of this session was to provide a space for young people to talk about death and improve coping strategies. The grief and loss session was reported to be helpful, but some younger children found the session traumatic. As psychosocial support addresses both primary (psychological) and secondary (social) outcomes, the sustainability of these interventions could increase. This is important to address as interventions can manage the effects of traumatic grief but could increase discrimination within the community.

Three interventions were identified that reported an improvement in social connectedness by increasing adult support within mentorship programs and improving peer relationships. Brown et $\mathrm{al}^{69}$ evaluated a mentorship program for young people aged 12-26 years in Rwanda who were doubly orphaned and heads of their households. This quasi-experimental study compared young people exposed to the mentorship program with those in a control group for a reduction in feelings of marginalization, grief, depression, and maltreatment. Mentoring involved regular home visits and the development of a therapeutic relationship. Findings revealed that adult mentorship mitigated the adverse psychosocial outcomes of orphanhood by reducing symptoms of depression $(P<0.009)$ and levels of marginalization $(P<0.004)$. The intervention did not significantly reduce the feelings of grief and maltreatment in comparison to the control group.

Kumakech et $\mathrm{al}^{70}$ evaluated a peer support group that assessed the influence of the intervention upon the psychosocial wellbeing of 10-year old and 15-year-old AIDS orphans in Uganda. Peer-group support involved young people participating in problem-solving exercises that related to specific issues in their lives. This intervention was found to be effective in decreasing psychological distress and reducing symptoms of depression, anxiety, and anger. Orphans who did not receive the intervention showed an increase in depression and anxiety. Denis and Makiwane ${ }^{37}$ evaluated the use of memory boxes, which involved members of families compiling stories and important objects pertaining to their history in a box before an anticipated death. This intervention provided an opportunity for families to talk about life, death, and plans for the future in order to promote resilience in young people at risk for experiencing traumatic grief. Creating memory boxes was found to be a helpful process to implement when preparing for bereavement. However, the session required active participation, which was variable across the study.

Kayombo et a ${ }^{35}$ evaluated the role of traditional healers in supporting orphans aged 3-19 years in Tanzania. Traditional healers live within communities at the grassroots level and are recognized for providing remedies for illnesses and diseases. In addition, those in this study were also caring for orphans of HIV/AIDS and even providing psychosocial support to help children cope with orphanhood. Psychological needs were met through the development of coping skills; social needs were addressed through acceptance and community integration; emotional needs were met through love, encouragement, and motivation; and physical needs were met through shelter, food, and school fees. The authors maintain that the role of traditional healers in caring for orphans needs to be recognized, and more resources should be provided to empower them and train them in basic psychosocial techniques so that orphans can be reintegrated into their communities without feeling stigmatized.

\section{Quality of formal and informal service provision reporting}

The intervention studies included in this paper (summarized in Table 2) that report existing services provisions were published between 2003 and 2010, implying research conducted within this field remains fairly recent. Despite the majority of interventions being carried out by local community providers, the findings have been published by authors who 


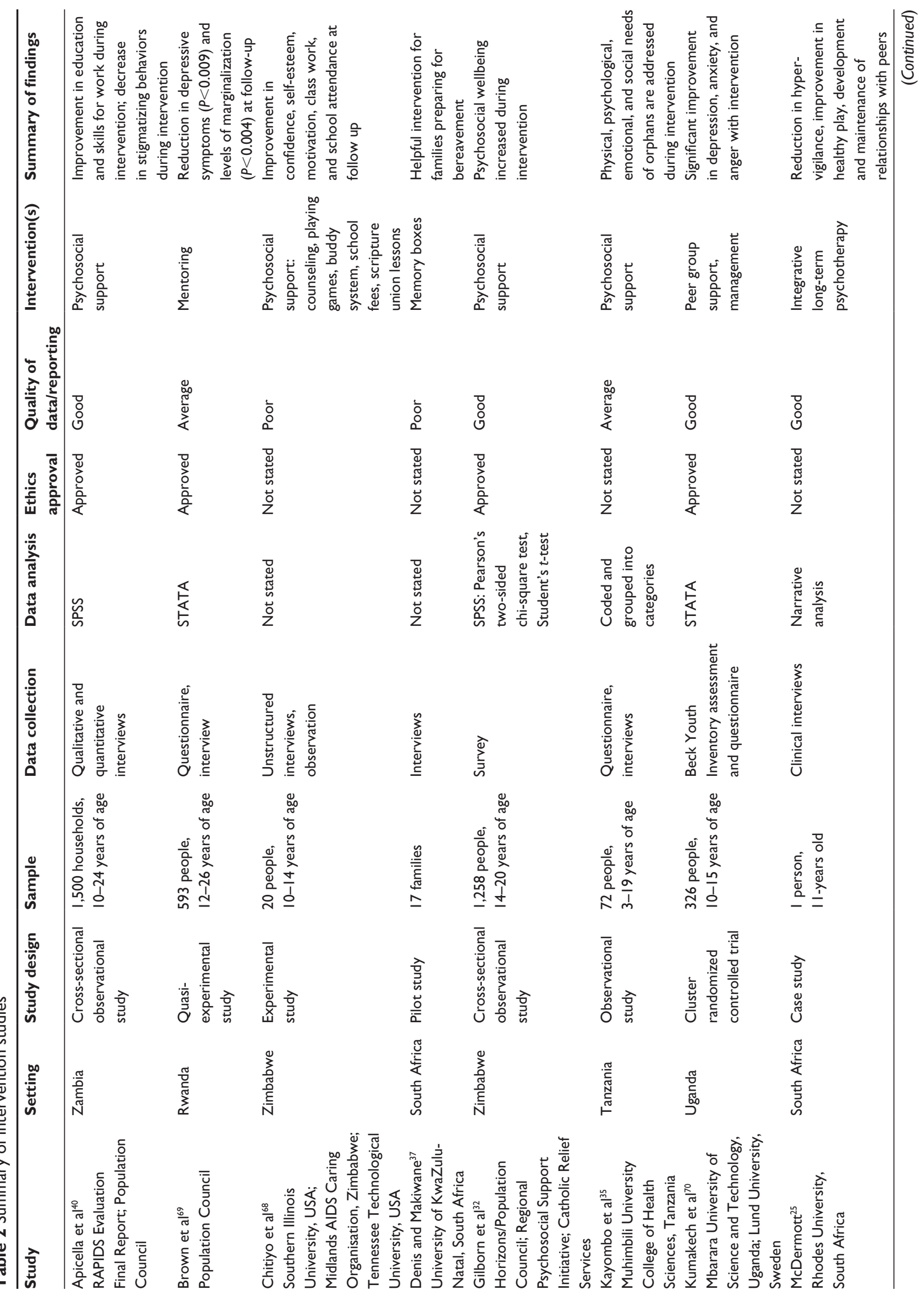




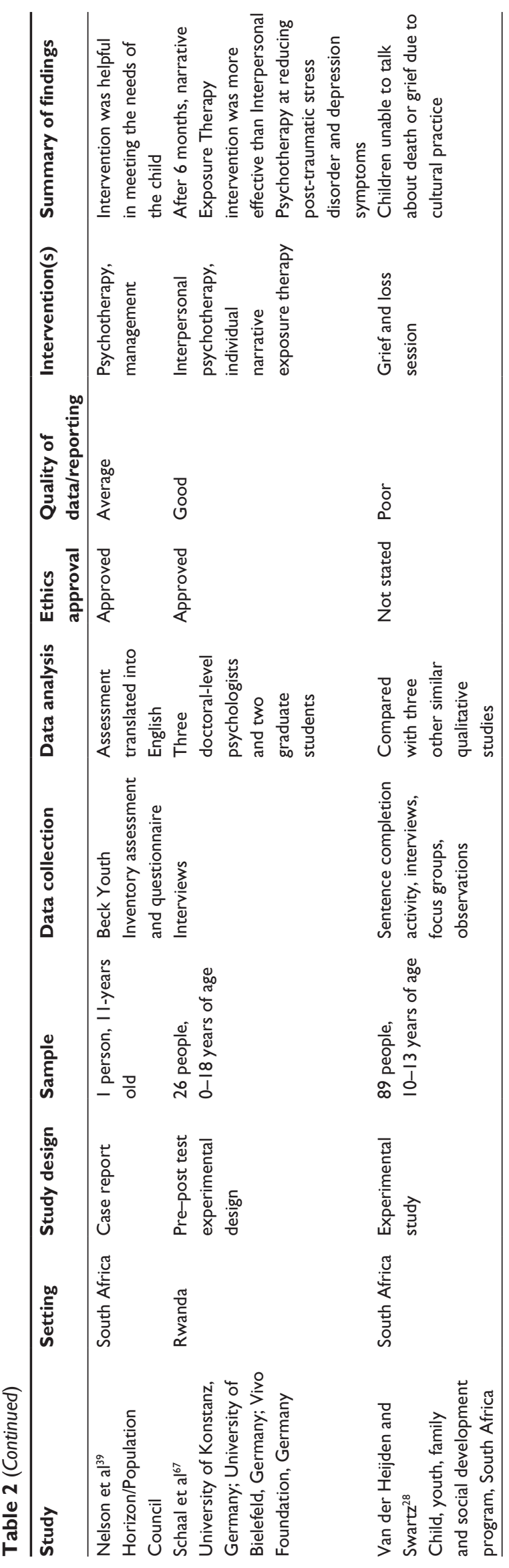

are affiliated with global charity organizations: none were published by authors from SSA. Kieling et $\mathrm{a}^{31}$ argue that health professionals in SSA ought to become more involved in service and policy development in order to take ownership of interventions within their communities; we would also urge them to lead and publish their research. This is important because the assessment of the success of an intervention from an outsider (etic) perspective may be different from a community (emic) perspective. Best practices do not appear to be shared between countries in SSA; few studies appeared to situate their work alongside that of others. This might be a consequence of the inaccessibility of health care information generally throughout SSA, but it highlights a need for professionals to communicate the effectiveness of interventions and transferable elements to other countries and cultures through publication and other sources. It is hoped that recent moves in open access information might facilitate this (eg, Health Information for All ${ }^{56}$ ).

With regard to the participants recruited to intervention studies, numbers ranged from 1 to 1,500 people and the age group most frequently cited was between 11 years and 14 years of age, perhaps suggesting that this age group are the most accessible and willing to participate. The majority of studies included both orphans and vulnerable young people and reported parental death as being a consequence of AIDS, indicating that this is the leading cause of orphanhood in SSA. Surprisingly, only six of the studies reported receiving ethical approval. The most common intervention implemented was psychosocial support, and the majority of interventions aimed to manage traumatic grief. Most interventions were delivered by members of the community as opposed to health or social care professionals, implying that mental health services are unable to meet the needs of communities or that community members are better placed to deliver these services. The reason for the lack of healthcare-based interventions could be the scarcity of skilled healthcare professionals. Within the mental health sector in SSA, there are only 0.05 psychiatrists, 0.61 nurses, and 0.04 psychologists for every 100,000 people. ${ }^{55}$ Resource limitations and the financial means to train staff can be significant barriers to service delivery. ${ }^{62} \mathrm{Maj}^{64}$ suggests that there is a need for psychiatrists to be trained to a higher standard, to cover a wider area of the population and to adhere to psychosocial interventions.

\section{Conclusion}

This scoping review has shown that CTG is an important topic within SSA and is highly relevant to the global mental 
health agenda. Although the rate of new HIV infections is said to be decreasing, the number of orphans has increased to unprecedented levels: SSA was said to account for $90 \%$ of the world's AIDS orphans in 2009. ${ }^{2}$ As the number of orphans rises, CTG in SSA is also set to rise. Despite this, the psychological health needs of adolescents are seldom recognized or adequately addressed in policy and programs. It is with this in mind that we present a number of recommendations developed from the reviewed literature that will help inform service development and future research priorities.

\section{Recommendations for service development}

- Educate service providers in identifying mental health problems through open-access training manuals/toolkits.

- Develop interventions/programs that prevent and/or manage traumatic grief in young people across countries throughout SSA, in order to increase service provisions and close the current gap.

- Design interventions/programs to meet the holistic (eg, material, physical, psychological, social, emotional, and educational) needs of young people.

- Create interventions/programs to prevent and/or manage the effects of a variety of psychological traumas (eg, violence, sexual abuse).

- Design interventions/programs to remain culturally sensitive (eg, single-sex groups, anonymity).

- Direct service providers and health professionals to tackle stigma and discrimination of HIV/AIDS, orphanhood, and mental illness.

- Direct service providers to increase awareness and promote interventions to members of the public and organizations.

- Encourage community providers to facilitate partnerships with health and social-care services for information sharing.

\section{Recommendations for research}

- Conduct a systematic review comparing specialist and nonspecialist interventions in SSA.

- Conduct observational research to ascertain why particular interventions are effective in certain contexts. This will identify cultural, environmental, political, and social factors and provide insight into transferability of interventions.

- Conduct longitudinal studies that aim to show associations between investment in child and adolescent mental health and improved child development, and reduced mental health problems in adulthood and improved economic productivity.

- Conduct qualitative studies to develop a greater understanding of the childhood experience of traumatic grief leading to interventions/programs tailored to the holistic needs of young people in SSA.

\section{Author contributions}

Both authors contributed toward data analysis, drafting and revising the paper and agree to be accountable for all aspects of the work.

\section{Disclosure}

The authors report no conflicts of interest in this work.

\section{References}

1. Melhem NM, Porta G, Shamseddeen W, Walker Payne M, Brent DA Grief in children and adolescents bereaved by sudden parental death. Arch Gen Psychiatry. 2011;68(9):911-919.

2. Joint United Nations Programme on HIV/AIDS (UNAIDS). UNAIDS Report on the Global Aids Epidemic 2010 [webpage on the Internet] Geneva, Switzerland: UNAIDS; 2010. Available from: http://www. unaids.org/globalreport/Global_report.htm. Accessed November 2, 2014.

3. Thupayagale-Tshweneagae G, Mokomane Z. Needs of South African adolescents orphaned by AIDS: evidence from photography and photo-elicitation. Int Nurs Rev. 2013;60(1):88-95.

4. Cluver L, Gardner F. The mental health of children orphaned by AIDS: a review of international and southern African research. JChild Adolesc Ment Health. 2007;19(1):1-17.

5. Thupayagale-Tshweneagae G, Wright SD, Hoffmann WA. Mental health challenges of the lived experiences of adolescents orphaned by HIV and AIDS in South Africa. J AIDS HIV Res. 2010;2(1):8-16.

6. Shatkin JP, Belfer ML. The global absence of child and adolescent mental health policy. Child Adolesc Ment Health. 2004;9(3):104-108.

7. The National Child Traumatic Stress Network. What is childhood traumatic grief? [webpage on the Internet]. Los Angeles, CA: National Center for Child Traumatic Stress; 2014 [updated April 20, 2014]. Available from: http://www.nctsn.org/trauma-types/traumatic-grief/ what-childhood-traumatic-grief. Accessed November 2, 2014.

8. United Nations. Composition of macro geographical (continental) regions, geographical sub-regions, and selected economic and other groupings [webpage on the Internet]. New York, NY: United Nations Publication Board; 2014 [updated October 31, 2013]. Available from: http://unstats.un.org/unsd/methods/m49/m49regin.htm. Accessed November 2, 2014.

9. Rumrill PD, Fitzgerald SM, Merchant WR. Using scoping literature reviews as a means of understanding and interpreting existing literature. Work. 2010;35(3):399-404.

10. Sandelowski M. "To be of use": enhancing the utility of qualitative research. Nurs Outlook. 1997;45(3):125-132.

11. Arksey H, O’Malley L. Scoping studies: towards a methodological framework. Int J Soc Res Methodol. 2005;8(1):19-32.

12. Levac D, Colquhoun H, O'Brien KK. Scoping studies: advancing the methodology. Implement Sci. 2010;5:69.

13. Cohen JA, Mannarino AP, Knudsen K. Treating childhood traumatic grief: a pilot study. JAm Acad Child Adolesc Psychiatry. 2004;43(10): 1225-1233.

14. Prigerson H. Complicated grief: when the path of adjustment leads to a dead-end. Bereave Care. 2004;23(3):38-40. 
15. Corr CA, Balk DE. Children's Encounters with Death, Bereavement, and Coping. New York, NY: Springer Publishing Company, LLC; 2010.

16. Beveridge A. On the origins of post traumatic stress disorder. In: Black D, Newman M, Harris-Hendriks J, Mezey G, editors. Psychological Trauma: A Developmental Approach. London, UK: The Royal College of Psychiatrists; 1997:3-9.

17. Terr LC. Childhood traumas: an outline and overview. Am J Psychiatry. 1991;148(1):10-20.

18. Scheeringa MS, Myers L, Putnam FW, Zeanah CH. Diagnosing PTSD in early childhood: an empirical assessment of four approaches. J Trauma Stress. 2012;25(4):359-367.

19. National Institute for Health and Care Excellence. Post-traumatic stress disorder (PTSD): The management of PTSD in adults and children in primary and secondary care. NICE guidelines [CG26] [webpage on the Internet]. London, UK: NICE; 2014. Available from: http://www.nice. org.uk/guidance/CG026. Accessed November 2, 2014.

20. Ross CA. Dissociation and psychosis: The need for integration of theory and practice. In: Johannessen JO, Martindale BV, Cullberg J, editors. Evolving Psychosis: Different Stages, Different Treatments. Hove, UK: Routledge; 2006:238-254.

21. Hammersley P, Bullimore P, Fiddler M, Read J. The trauma model of psychosis. In: Stickley T, Basset T, editors. Learning About Mental Health Practice. Chichester, UK: John Wiley \& Sons Ltd; 2008:401-416.

22. Newman E, Kaloupek D. Overview of research addressing ethical dimensions of participation in traumatic stress studies: autonomy and beneficence. J Trauma Stress. 2009;22(6):595-602.

23. Sikkema KJ, Hansen NB, Kochman A, Tate DC, Difranceisco W. Outcomes from a randomized controlled trial of a group intervention for HIV positive men and women coping with AIDS-related loss and bereavement. Death Stud. 2004;28(3):187-209.

24. Regel S, Joseph S. Post-traumatic Stress (The Facts). Oxford, UK: Oxford University Press; 2010.

25. McDermott R. The great arch of unimagined bridges: integrative play therapy with an abused child. J Psychol Afr. 2005;15(2):165-175.

26. Wood K, Chase E, Aggleton P. 'Telling the truth is the best thing': teenage orphans' experiences of parental AIDS-related illness and bereavement in Zimbabwe. Soc Sci Med. 2006;63(7):1923-1933.

27. Withell B. The prebereavement psychological needs of AIDSaffected adolescents in Uganda. Int J Palliat Nurs. 2009;15(3): $128-133$.

28. Van der Heijden I, Swartz S. Bereavement, silence and culture within a peer-led HIV/AIDS-prevention strategy for vulnerable children in South Africa. Afr J AIDS Res. 2010;9(1):41-50.

29. Auman MJ. Bereavement support for children. J Sch Nurs. 2007;23(1): 34-39.

30. Masten AS. Ordinary magic. Resilience processes in development. Am Psychol. 2001;56(3):227-238.

31. Kieling C, Baker-Henningham $\mathrm{H}$, Belfer M, et al. Child and adolescent mental health worldwide: evidence for action. Lancet. 2011;378(9801): 1515-1525.

32. Gilborn LZ, Apicella L, Brackarsh J, et al. Orphans and Vulnerable Youth in Bulawayo, Zimbabwe: An Exploratory Study of Psychosocial Well-being and Psychosocial Support Programs. Horizons Final Report. Washington, DC: Population Council; 2006.

33. Gilborn LZ, Nyonyintono R, Kabumbuli R, Jagwe-Wadda G. Making a Difference for Children Affected by AIDS: Baseline Findings from Operations Research in Uganda. New York, NY: Population Council; 2001.

34. Onuoha FN, Munakata T, Serumaga-Zake PA, Nyonyintono RM, Bogere SM. Negative mental health factors in children orphaned by AIDS: natural mentoring as a palliative care. AIDS Behav. 2009;13(5): 980-988.

35. Kayombo EJ, Mbwambo ZH, Massila M. Role of traditional healers in psychosocial support in caring for the orphans: a case of Dar-es Salaam City, Tanzania. J Ethnobiol Ethnomed. 2005;1:3.
36. Makame V, Ani C, Grantham-McGregor S. Psychological well-being of orphans in Dar El Salaam, Tanzania. Acta Paediatr. 2002;91(4): 459-465.

37. Denis P, Makiwane N. Stories of love, pain and courage: AIDS orphans and memory boxes. Oral Hist (Colch). 2003;31(2):66-74.

38. World Health Organization. mhGAP Intervention Guide for Mental, Neurological and Substance Abuse Disorders in Non-Specialized Health Settings. Geneva, Switzerland: World Health Organization; 2010. Available from: http://whqlibdoc.who.int/publications/2010/9789241548069_eng. pdf?ua=1. Accessed November 2, 2014.

39. Nelson TC, Esu-Williams E, Mchunu L, Nyamakazi P, Mnguni S. Training Youth Caregivers to Provide HIV Education and Support to Orphans and Vulnerable Children in South Africa. Horizons Research. Washington, DC: Population Council; 2008.

40. Apicella L, Schenk K, Khan H. RAPIDS Evaluation Final Report 2005-2009 Key Findings. Lusaka, Zambia: Population Council; 2010. Available from: http://www.popcouncil.org/uploads/pdfs/2010HIV_ RAPIDSEval.pdf. Accessed May 6, 2015

41. Idoniboye G. A call for mental health needs assessments in HIV positive children in Africa. J R Soc Promot Health. 2008;128(5): 240-241.

42. Cluver L, Gardner F. Risk and protective factors for psychological well-being of children orphaned by AIDS in Cape Town: a qualitative study of children and caregivers' perspectives. AIDS Care. 2007;19(3): 318-325.

43. Cluver L, Orkin M. Cumulative risk and AIDS-orphanhood: interactions of stigma, bullying and poverty on child mental health in South Africa. Soc Sci Med. 2009;69(8):1186-1193.

44. Robben ACGM. Death, Mourning and Burial: A Cross-Cultural Reader. Oxford, UK: Blackwell Publishing, Ltd; 2004.

45. Opperman BCD. The Influence of Context on Complicated Grief [master's thesis]. Johannesburg, South Africa: Rand Afrikaans University; 2004

46. Prigerson HG, Maciejewski PK, Reynolds CF, et al. Inventory of Complicated Grief: a scale to measure maladaptive symptoms of loss. Psychiatry Res. 1995;59(1-2):65-79.

47. Melhem NM, Day N, Shear MK, Day R, Reynolds CF, Brent D. Traumatic grief among adolescents exposed to a peer's suicide. Am J Psychiatry. 2004;161(8):1411-1416.

48. American Psychiatric Association. Highlights of Changes from DSM$I V-T R$ to DSM-5. Arlington, VA: American Psychiatric Association; 2013. Available from: http://www.dsm5.org/Documents/changes from dsm-iv-tr to dsm-5.pdf. Accessed November 2, 2014.

49. Bisson JI, Brayne M, Ochberg FM, Everly GS. Early psychosocial intervention following traumatic events. Am J Psychiatry. 2007;164(7): 1016-1019.

50. Dyregrov A. The process in psychological debriefings. JTrauma Stress. 1997;10(4):589-605.

51. Kassam-Adams N. The Acute Stress Checklist for Children (ASCKids): development of a child self-report measure. J Trauma Stress. 2006;19(1):129-139.

52. Foa EB, Johnson KM, Feeny NC, Treadwell KR. The child PTSD symptom scale: a preliminary examination of its psychometric properties. J Clin Child Psychol. 2001;30(3):376-384.

53. Ehlers A, Clark DM. A cognitive model of posttraumatic stress disorder. Behav Res Ther. 2000;38(4):319-345.

54. Coetzee RH, Regel S. Eye movement desensitisation and reprocessing: an update. Adv Psychiatr Treat. 2005;11(5):347-354.

55. World Health Organization. Mental Health Atlas 2011 [webpage on the Internet]. Geneva, Switzerland: World Health Organization; 2011. Available from: http://www.who.int/mental_health/publications/ mental_health_atlas_2011/en/.Accessed November 2, 2014.

56. Healthcare Information for All (HIFA). A global campaign: Healthcare Information For All by 2015 [homepage on the Internet]. HIFA. Oxfordshire, UK: Global Healthcare Information Network; 2015. Available from: http://www.hifa2015.org. Accessed November 2, 2014 
57. United Nations. The Millennium Development Goals Report, 2010. New York, NY: United Nations Department of Economic and Social Affairs; 2010. Available from: http:/www.un.org/millenniumgoals/ pdf/MDG\%20Report\%202010\%20En\%20r15\%20-low\%20res\%20 20100615\%20-.pdf. Accessed November 2, 2014.

58. Eaton J, McCay L, Semrau M, et al. Scale up of services for mental health in low-income and middle-income countries. Lancet. 2011; 378(9802):1592-1603.

59. Okasha A. Mental health in Africa: the role of the WPA. World Psychiatry. 2002;1(1):32-35.

60. Patel V, Boyce N, Collins PY, Saxena S, Horton R. A renewed agenda for global mental health. Lancet. 2011;378(9801):1441-1442.

61. Buchan J, Calman L. Summary. The Global Shortage of Registered Nurses: An Overview of Issues and Actions. Geneva, Switzerland: International Council of Nurses; 2004. Available from: http://www.icn. ch/images/stories/documents/publications/GNRI/Global_Shortage_of_ Registered_Nurses_Executive_summary.pdf. Accessed November 2, 2104.

62. Crisp N. Global Health Partnerships. The UK Contribution to Health in Developing Countries. London, UK: Tropical Health and Education Trust; 2007. Available from: http://www.thet.org/hps/resources/publications-old/lord-crisp-report-2007-1. Accessed: November 2, 2014.

63. World Health Organization. The World Health Report 2006. Chapter 1: Health Workers: A Global Profile. Geneva, Switzerland: World Health Organization; 2006. Available from: http://www.who.int/whr/2006/06 chap1_en.pdf?ua=1. Accessed November 2, 2014.
64. Maj M. The rights of people with mental disorders: WPA perspective. Lancet. 2011;378(9802):1534-1535.

65. Gerein N, Green A, Pearson S. The implications of shortages of health professionals for maternal health in sub-saharan Africa. Reprod Health Matters. 2006;14(27):40-50.

66. Barrett T, Boeck R, Fusco C, Ghebrehiwet T, Yan J, Saxena S. Nurses are the key to improving mental health in low- and middle-income countries. Int Nurs Rev. 2009;56(1):138-141.

67. Schaal S, Elbert T, Neuner F. Narrative exposure therapy versus interpersonal psychotherapy. A pilot randomized controlled trial with Rwandan genocide orphans. Psychother Psychosom. 2009;78(5): 298-306.

68. Chitiyo M, Changara DM, Chitiyo G. Providing psychosocial support to special needs children: A case of orphans and vulnerable children in Zimbabwe. Int J Educ Dev. 2008;28(4):384-392.

69. Brown L, Rice J, Boris N, et al. Psychosocial Benefits of a Mentoring Program for Youth-Headed Households in Rwanda. Horizons Research Summary. Washington, DC: Population Council; 2007. Available from: http://54.172.146.51/sites/default/files/g3p_documents/68/HorizonsResearch-Study-Summary.pdf. Accessed May 6, 2015.

70. Kumakech E, Cantor-Graae E, Maling S, Bajunirwe F. Peer-group support intervention improves the psychosocial well-being of AIDS orphans: cluster randomized trial. Soc Sci Med. 2009;68(6):1038-1043.
Nursing: Research and Reviews

\section{Publish your work in this journal}

Nursing: Research and Reviews is an international, peer-reviewed, open access journal publishing original research, reports, reviews and commentaries on all aspects of nursing and patient care. These include patient education and counselling, ethics, management and organizational issues, diagnostics and prescribing, economics and

\section{Dovepress}

resource management, health outcomes, and improving patient safety in all settings. The manuscript management system is completely online and includes a very quick and fair peer-review system. Visit http://www.dovepress.com/testimonials.php to read real quotes from published authors. 